\title{
Artificial-Intelligence Method for the Derivation of Generic Aggregated Dynamic Equivalent Models
}

\author{
Eleftherios O. Kontis, Student Member, IEEE, Theofilos A. Papadopoulos, Senior Member, IEEE, \\ Mazheruddin H. Syed, Member, IEEE, Efren Guillo-Sansano, Student Member, IEEE, \\ Graeme M. Burt, Member, IEEE, and Grigoris K. Papagiannis, Senior Member, IEEE
}

\begin{abstract}
Aggregated equivalent models for the dynamic analysis of active distribution networks (ADNs) can be efficiently developed using dynamic responses recorded through field measurements. However, equivalent model parameters are highly affected from the time-varying composition of power system loads and the stochastic behavior of distributed generators. Thus, equivalent models, developed through in-situ measurements, are valid only for the operating conditions from which they have been derived. To overcome this issue, in this paper, a new method is proposed for the derivation of generic aggregated dynamic equivalent models, i.e., for equivalent models which can be used for the dynamic analysis of a wide range of network conditions. The method incorporates clustering and artificial neural network techniques to derive robust sets of parameters for a variable-order dynamic equivalent model. The effectiveness of the proposed method is evaluated using measurements recorded on a laboratory-scale ADN, while its performance is compared with a conventional technique. The corresponding results reveal the applicability of the proposed approach for the analysis and simulation of a wide range of distinct network conditions.
\end{abstract}

Index Terms-Artificial Neural Networks, black-box modeling, clustering, dynamic modeling, measurement-based approach.

\section{INTRODUCTION}

$\mathbf{T}$ HE advent of microgrids (MGs) and the increased penetration of distributed generators (DGs) into the existing distribution grids have changed drastically the dynamic properties of power systems [1]-[3]. Under these new operating conditions, academia and power system operators have initiated serious efforts to develop accurate and adaptive dynamic simulation models to enhance the analysis of modern active distribution networks (ADNs) [3] as well as to investigate more efficient modes of operation for the DGs.

Manuscript received June, 2018, revised October and December, 2018.

E. O. Kontis and G. K. Papagiannis are with the Power Systems Laboratory, School of Electrical and Computer Engineering, Aristotle University of Thessaloniki, Thessaloniki, Greece, GR 54124, (e-mail: ekontis@auth.gr; grigoris@eng.auth.gr).

T. A. Papadopoulos is with the Power Systems Laboratory, Department of Electrical and Computer Engineering, Democritus University of Thrace, Xanthi, Greece, GR 67100, (e-mail: thpapad@ee.duth.gr).

M. H. Syed, E. Guillo-Sansano, and G. M. Burt are with the Institute for Energy and Environment, University of Strathclyde, Glasgow, UK, (e-mail: mazheruddin.syed@strath.ac.uk; efren.guillo-sansano@strath.ac.uk; graeme.burt@strath.ac.uk).

The work of E. O. Kontis was conducted in the framework of the act "Support of $\mathrm{PhD}$ Researchers" under the Operational Program "Human Resources Development, Education and Lifelong Learning 2014-2020", which is implemented by the State Scholarships Foundation and co-financed by the European Social Fund and the Hellenic Republic.

The work in this paper has been supported by the European Commission, under the FP7 project ELECTRA (grant no: 609687) and under H2020 project ERIGRID (grant no:654113).
In the literature, there are several publications using detailed models to investigate the dynamic properties of ADNs and MGs [4]-[6]. However, the development of detailed dynamic models requires very accurate information concerning the structure of the examined grid and the control parameters of the installed DGs. Therefore, this approach requires accurate data, which cannot be determined in distribution networks due to their extended size. Additionally, it is worth noticing that this approach requires significant computational resources, leading also to large simulation times [1].

To reduce the computational burden of dynamic simulations, reduced order dynamic equivalent models have been proposed. The work on this field can be classified into three main approaches [7]. The first one contains coherency-based methods, where group of coherent generators are identified and replaced by equivalent generators. In the second methodology approximate linear models of the examined system are derived using modal analysis techniques. However, in both approaches, the identification of model parameters requires detailed network information, thus the drawbacks and restrictions of detailed modeling apply to these methods as well [8].

A promising option to overcome the lack of detailed information is to derive gray- or black-box dynamic equivalent models using the third methodology, i.e., the measurementbased approach [1]. In this case, model parameters are identified from field measurements, by applying system identification techniques [7]. However, parameters of measurementbased equivalents are highly affected from the weatherdependent and stochastic behavior of DGs as well as from the time-varying composition of power system loads [9]-[11]. Thus, model parameters are valid only for the operating condition from which they have been derived and cannot be used to simulate accurately different cases [9], [12], i.e., different disturbances, network configurations, loading conditions, etc.

To determine robust model parameters for measurementbased dynamic equivalent models, several approaches have been proposed. In [1], [2] and [10], statistical analysis is applied and the required model parameters are identified by calculating the corresponding median or mean values. Multisignal analysis techniques are developed in [9], [13] and [14], while in [8] and [15], linear approximation functions are proposed. However, in all the above-mentioned approaches, human interaction is always required to divide the available data sets into groups, presenting similar characteristics (e.g. data groups obtained under similar network conditions [9], [10]). Additionally, it is worth noticing that using the above 
techniques, model parameters that are valid only for a narrow range of network conditions can be estimated [1], [9].

To develop generic equivalent models, able to account for a wide range of network conditions, the use of artificialintelligence techniques have been proposed in the literature. In [16] and [17], dynamic equivalent models based on recurrent artificial neural networks (ANNs) and radial basis functions ANNs are proposed. However, the parameters of these models have no physical meaning [18], thereby offering limited insight to power system engineers concerning dynamic properties of the grid. To address this issue, in [12], [19], [20] artificial intelligence techniques are proposed to derive generic parameters for conventional equivalent models. More specifically, in [19] support vector clustering is proposed to derive generic parameters for a transfer function-based equivalent model, while in [12] and [20] the use of ANNs is proposed to generalize the parameters of power system load models. However, these methods present certain shortcomings and limitations. For instance, the method proposed in [19] requires measurements from all ADN feeders to provide consistent results, while the approaches of [12] and [20] are focused on conventional load models [21], which cannot describe effectively the dynamic behavior of modern ADNs [1], [22], [23]. Moreover, the performance of the above approaches has only been tested using simulation results. Thus, their applicability for real field applications still remains an open issue.

Considering the above issues, the primary scope of the paper is to develop a new method for the derivation of generic measurement-based equivalent models, suitable for the dynamic analysis of modern ADNs and for the simulation of a wide range of distinct network conditions. The second objective is to evaluate the performance of the proposed method using laboratory measurements.

The proposed method receives as inputs the operating conditions (i.e. voltage level, real and reactive power consumption or production), the load and the generation mix of the examined ADN. Using these inputs, an aggregated ADN model is automatically developed, describing efficiently the dynamic behavior of the examined ADN. To fulfill this objective, clustering and ANN techniques are used to derive robust sets of parameters for the variable-order aggregated equivalent model of [22]. This model structure is selected as it allows the simulation of bi-directional power flow phenomena which may occur in modern ADNs during voltage events [22] as well as the simulation of complex power system dynamics, occurring after small or large system disturbances [14]. Additionally, the parameters of this model have a strong physical meaning [14].

The effectiveness of the proposed method is evaluated under various network configurations, load and generation mixes, operating conditions and voltage disturbances, using measurements acquired from a laboratory-scale ADN. Additionally, its performance is compared with a conventional approach, in which robust sets of parameters are determined based on mean characteristics, by applying statistical analysis.

\section{Dynamic Equivalent Model}

The dynamic response of a distribution network subjected to a step-down voltage disturbance (Fig. 1a) is presented in
Fig. 1b. As shown, immediately after the disturbance, the power demand decreases instantaneously to $y_{+}$value. After this transient undershoot a recovery phase occurs and the power gradually recovers to the new steady-state value, i.e. $y_{s s}$. This dynamic behavior can be accurately simulated using the aggregated equivalent model of [22]. The block diagram representation of this model is depicted in Fig. 2, while its mathematical formulation is given in the following equations:

$$
y_{d}(t)=y_{t}(t)+y_{r}(t)
$$

where

$$
\begin{gathered}
y_{r}(t)=\mathcal{L}^{-1}\left[g_{2}(s) G(s)\right] \\
y_{t}(t)=y_{0}\left[\lambda_{1}\left(\frac{V_{L}(t)}{V_{0}}\right)+\lambda_{2}\right], \quad \sum_{i=1}^{2} \lambda_{i}=1 \\
y_{s}(t)=y_{0}\left[\kappa_{1}\left(\frac{V_{L}(t)}{V_{0}}\right)+\kappa_{2}\right], \quad \sum_{i=1}^{2} \kappa_{i}=1 \\
g_{1}(t) \equiv y_{t}(t), \quad g_{2}(t) \equiv y_{s}(t)-y_{t}(t) \\
G(s)=\sum_{i=1}^{n} \frac{c_{i}}{s-p_{i}}
\end{gathered}
$$

Here, $y_{d}(t)$ can represent both real and reactive power responses. Functions $y_{t}(t)$ and $y_{s}(t)$ are two polynomial functions, used to simulate the transient and the steadystate response of the examined ADN [22], [23], respectively. Moreover, $\lambda_{1}, \lambda_{2}$ and $\kappa_{1}, \kappa_{2}$ are the polynomial coefficients of $y_{t}$ and $y_{s}$, respectively [22], [23]. The recovery response of the power, i.e. $y_{r}(t)$, is approximated using functions $g_{2}(s)$ and $G(s)$ [14], [22]. $g_{2}(s)$ denotes the Laplace transform of $g_{2}(t)$, while $G(s)$ is a variable-order linear transfer function [14], [22]. $\boldsymbol{p}$ and $\boldsymbol{c}$ stand for the poles and residues of $G(s)$, respectively, while $n$ denotes the optimal order of $G(s)$. The optimal order $n$ can be determined automatically by applying the iterative procedure proposed in [22]. Finally, $V_{L}(t)$ is the ADN voltage, whereas $y_{0}$ and $V_{0}$ are the power and voltage magnitude prior to the examined disturbance.
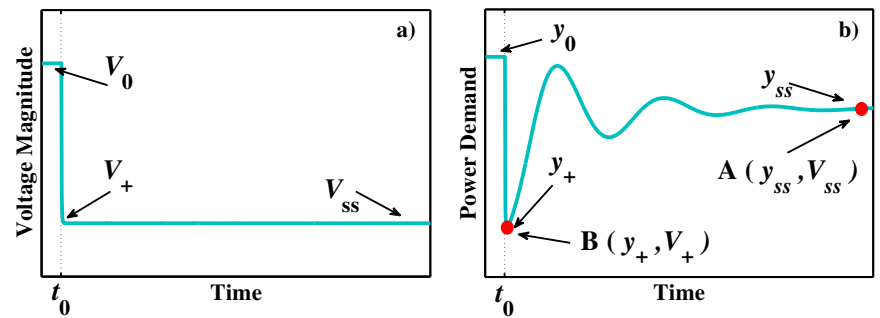

Fig. 1. a) Indicative step-down voltage disturbance. b) Representative real or reactive power response.

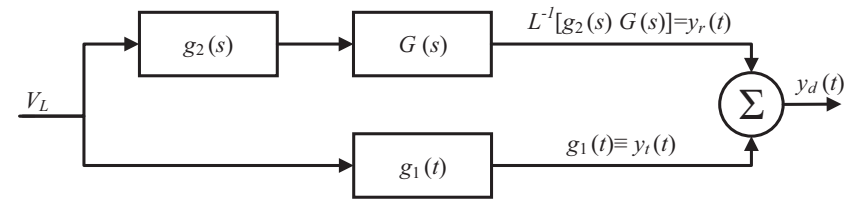

Fig. 2. Block diagram for the integration of the adopted dynamic equivalent model into simulation software. 


\section{Proposed Generic Modeling ApProACH}

The procedure used to develop the proposed modeling approach is conceptually summarized in Fig. 3. Initially, a database is developed, containing $N$ data sets. In each data set, the following variables are stored: three vectors containing the measured dynamic responses (i.e. RMS values of timedomain signals) of i) voltage $\left(\boldsymbol{V}_{i}\right)$, ii) real $\left(\boldsymbol{P}_{i}\right)$ and iii) reactive power $\left(\boldsymbol{Q}_{i}\right)$. These responses reflect the dynamic behavior of the ADN during the $i$-th disturbance $(i=1, \ldots, N)$ and can be recorded at the point of common coupling (PCC) with the external grid using phasor measurement units (PMUs) [1], [2]. Moreover, two additional variables, namely the iv) load $\left(L M_{i}\right)$ and v) generation mix $\left(G M_{i}\right)$ are used. These variables reflect the load composition and the type of the installed DG units, respectively. The values of $L M_{i}$ and $G M_{i}$ can be assessed through forecasts or smart meter recordings [24]-[26].

Once the database has been developed, a pre-processing phase is applied. During this phase, the pre-disturbance steadystate values of voltage $\left(V_{0, i}\right)$, real $\left(P_{0, i}\right)$, and reactive power $\left(Q_{0, i}\right)$ are derived for all the available $N$ data sets. This information is used to provide an insight of the ADN operating conditions prior to the examined disturbance. Additionally, all dynamic responses are normalized, using the corresponding pre-disturbance steady-state values. Afterwards, the available $N$ data sets are randomly split into two separate groups, consisting of $N_{D}$ and $N_{T}$ data sets, respectively. The first group is used to develop generic equivalent models, suitable for the analysis of a wide range of network conditions, while the remaining $N_{T}$ data are used to test the performance of the derived models. The procedures, to develop and test the derived models, are depicted in Figs. $4 \mathrm{a}$ and $4 \mathrm{~b}$, respectively.

As shown in Fig. 4a, the proposed method consists of three main stages: i) clustering of available data, ii) parameter estimation, and iii) training of ANNs. The objective of the clustering is to automatically (i.e. without human interaction) divide the available data into $K$ groups, presenting similar pre-disturbance operating conditions. Then, for each cluster, a multi-signal identification procedure is performed to estimate the model parameters that optimally simulate the dynamic behavior of the ADN. Finally, for each cluster, an ANN is developed. Scope of the ANN is to capture general relationships between the model parameters and the examined network conditions. Using this information, robust sets of parameters are derived. A detailed explanation is presented below.

\section{A. Stage 1: k-means ++ Clustering Algorithm}

At this stage, clustering is applied to automatically divide the available data into $K$ groups, that present similar characteristics. The clustering algorithm, used in this paper, is the $k$-means++, an algorithm of proved efficiency for a wide range of applications [27], [28].

In the proposed framework, voltage magnitude, real and reactive power flows at the $\mathrm{PCC}$ are monitored by distribution system operator (DSO), using PMU devices [29]. On the other hand, load and generation mix (i.e. variables $L M$ and $G M$ ) of the ADN are estimated by the DSO based on forecasts or smart meter recordings. Therefore, in case of forecasts errors,

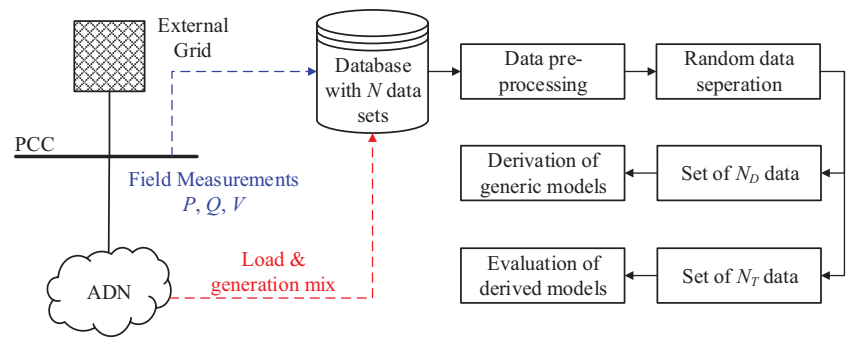

Fig. 3. Conceptual description of the proposed method.

errors in the values of these variables may be observed. Thus, to reduce the impact of forecast errors in the accuracy of the proposed method, variables $L M$ and $G M$ are neglected from the clustering procedure. Hence, the clustering is performed based only on the pre-disturbance operating conditions of the ADN, i.e. based on $V_{0, j}, P_{0, j}$, and $Q_{0, j}$ (here $j=1, \ldots, N_{D}$ ). To apply the clustering, $V_{0, j}, P_{0, j}$, and $Q_{0, j}$ are combined, for each of the available $N_{D}$ data sets, into one single vector $\boldsymbol{X}_{j}=\left[V_{0, j}, P_{0, j}, Q_{0, j}\right]$. Thus, a set $\boldsymbol{X}$ of $N_{D}$ observations $\boldsymbol{X}=\left\{\boldsymbol{X}_{1}, \boldsymbol{X}_{2}, \ldots, \boldsymbol{X}_{N D}\right\}$ is formed (where each observation is a $d$ dimensional vector) and forwarded as input to the $k$-means++ algorithm. Then, the $k$-means++ algorithm clusters the $N_{D}$ available data sets into $K\left(\leq N_{D}\right)$ clusters $\boldsymbol{C}=\left\{C_{1}, C_{2}, \ldots, C_{K}\right\}$ in order to minimize the within cluster sum of squares [28], as shown in (7).

$$
\underset{\boldsymbol{C}}{\arg \min } \sum_{k=1}^{K} \sum_{\boldsymbol{X}_{\boldsymbol{j}} \in C}\left\|\boldsymbol{X}_{\boldsymbol{j}}-\boldsymbol{\mu}_{\boldsymbol{k}}\right\|^{2}
$$

Where $\boldsymbol{\mu}_{\boldsymbol{k}}$ is the mean value of the points in the $k$-th cluster. Each cluster of data is represented by its centroid, which defines a representative location in the $d$ dimensional space for all the members of that particular cluster. To derive the optimal number (i.e. $K$ ) of clusters, the knee-point criterion for the curve of the Within Cluster sum of squares to Between Cluster sum variation Ratio (WCBCR) is used [30]. According to this criterion, the optimal number of clusters is defined by the knee of the curve [30].

\section{B. Stage 2: Parameter Estimation}

At this stage, the dynamic responses of voltage, real and reactive power, i.e. vectors $\boldsymbol{V}_{j}, \boldsymbol{P}_{j}$ and $\boldsymbol{Q}_{j}$, are used to estimate the model parameters, i.e. $\boldsymbol{\theta}_{j}=\left[\kappa_{1}, \kappa_{2}, \lambda_{1}, \lambda_{2}, \boldsymbol{p}, \boldsymbol{c}\right]$. Assuming a set of $m$ data sets for the $k$-th cluster, the following multi-signal analysis is performed:

Step 1: For each one of the $m$ data sets, parameters $\kappa_{1}, \kappa_{2}$, $\lambda_{1}$, and $\lambda_{2}$ are identified from operating points (please refer to Fig. 1) $A\left(y_{s s}, V_{s s}\right)$ and $B\left(y_{+}, V_{+}\right)$, respectively, using the following equations [22]:

$$
\begin{array}{rlrl}
\kappa_{1} & =\left[V_{0}\left(y_{s s}-y_{0}\right)\right] /\left[y_{0}\left(V_{s s}-V_{0}\right)\right], & & \kappa_{2}=1-\kappa_{1} \\
\lambda_{1} & =\left[V_{0}\left(y_{+}-y_{0}\right)\right] /\left[y_{0}\left(V_{+}-V_{0}\right)\right], & \lambda_{2}=1-\lambda_{1}
\end{array}
$$

here, $V_{+}$and $V_{s s}$ are voltage magnitude at PCC immediately after the disturbance and at the new steady-state, respectively.

Step 2: The polynomial functions $g_{1}$ and $g_{2}$ are computed for all the available $m$ data sets, using Eqn. (5). 


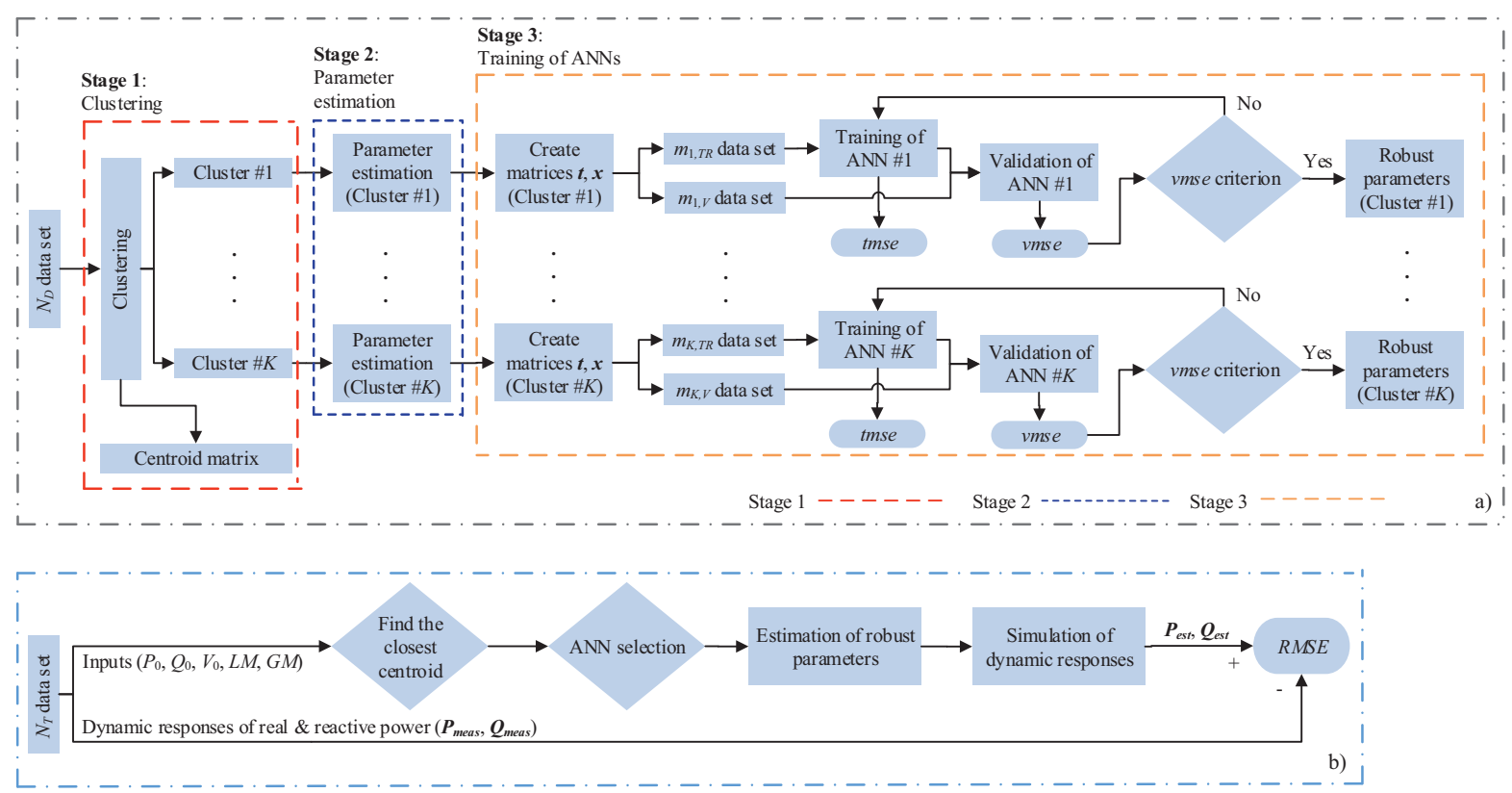

Fig. 4. Flowchart of the proposed modeling approach. a) Procedure for the derivation of generic dynamic equivalent models and b) procedure used to test the performance of the derived models.

Step 3: Here, (1) and (2) are used and $m$ individual responses are derived for the $G(s)$.

Step 4: The $m$ distinct responses of $G(s)$ are grouped together and inserted as inputs to the Vector Fitting (VF) algorithm [14], [31]. Using the VF, the $m$ distinct $G(s)$ responses are approximated via a common set of poles and $m$ distinct set of residues. The use of a common set of poles implies that all responses, belong at the same cluster, are approximated using a model of the same order, i.e., the value of parameter $n$ is common for all $m$ responses. Therefore, following this approach, only the dominant system modes are included in the developed model [32]. A detailed analysis of the parameter estimation procedure can be found in [14].

\section{Stage 3: Derivation of Robust Parameters using ANNs}

Scope of this stage is to generalize the parameters of the equivalent model in order to extend its applicability for the analysis of a wide range of network conditions, i.e. for network conditions different from those it has been originally developed using the available training data.

In this paper, the two-layer feed-forward ANN of Fig. 5 is used for the generalization of the model parameters. The proposed ANN consists of a hidden and an output layer. Each layer contains [33]: an input matrix $\boldsymbol{x}$, a weight matrix $\boldsymbol{W}$, a bias matrix $\boldsymbol{b}$, a sum operator, a transfer function $f$, denoted as $\mathrm{TF}$, and the output matrix $\boldsymbol{y}$. The weighting matrix weights the input elements, while the bias vector biases the corresponding weighted inputs. The sum operator gathers the biases and the weighted inputs and generates an intermediate variable for the associated TF. The TF produces the final outcome of the layer [33]. The input/output relationship in both the output and the hidden layer can be represented as:

$$
\boldsymbol{y}=f\left(\boldsymbol{W}^{T} \boldsymbol{x}+\boldsymbol{b}\right)
$$

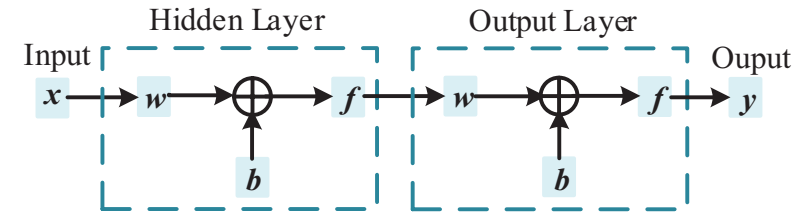

Fig. 5. Structure of the proposed ANN.

The inputs $(\boldsymbol{x})$ of the proposed ANN include: the predisturbance steady-state values of voltage, real and reactive power at the PCC as well as the load and the generation mix of the examined ADN. The targets $(t)$ are the corresponding equivalent model parameters. $\boldsymbol{x}$ and $\boldsymbol{t}$ can be written as:

$$
\begin{aligned}
& \boldsymbol{x}=\left[\begin{array}{ccc}
P_{0,1} & \ldots & P_{0, m_{k, T R}} \\
Q_{0,1} & \ldots & Q_{0, m_{k, T R}} \\
V_{0,1} & \ldots & V_{0, m_{k, T R}} \\
L M_{1} & \ldots & L M_{m_{k, T R}} \\
G M_{1} & \ldots & G M_{m_{k, T R}}
\end{array}\right] \\
& \boldsymbol{t}=\left[\begin{array}{ccc}
\kappa_{1,1} & \ldots & \kappa_{1, m_{k, T R}} \\
\lambda_{1,1} & \ldots & \lambda_{1, m_{k, T R}} \\
\boldsymbol{c}_{1} & \ldots & \boldsymbol{c}_{m_{k, T R}}
\end{array}\right]
\end{aligned}
$$

where $m_{k, T R}$ denotes the number of data sets that belong to the $k$-th cluster and are used for the training of the corresponding ANN. Note that the following set of parameters: $\boldsymbol{\theta}_{\boldsymbol{\kappa}_{\mathbf{2}}}=\left[\kappa_{2,1}, \ldots, \kappa_{2, m_{k, T R}}\right], \boldsymbol{\theta}_{\boldsymbol{\lambda}_{\mathbf{2}}}=\left[\lambda_{2,1}, \ldots, \lambda_{2, m_{k, T R}}\right]$, and $\boldsymbol{p}$ are not included in the target matrix. The latter is omitted, since all dynamic responses contained in a specific cluster are approximated using a common set of poles. On the other hand, $\boldsymbol{\theta}_{\boldsymbol{\kappa}_{2}}$ and $\boldsymbol{\theta}_{\boldsymbol{\lambda}_{2}}$ can be directly computed from (8) and (9) using the values of $\boldsymbol{\theta}_{\boldsymbol{\kappa}_{1}}=\left[\kappa_{1,1}, \ldots, \kappa_{1, m_{k, T R}}\right]$ and $\boldsymbol{\theta}_{\boldsymbol{\lambda}_{\mathbf{1}}}=\left[\lambda_{1,1}, \ldots, \lambda_{1, m_{k, T R}}\right]$, respectively. Following this approach, the size of the target matrix is considerably reduced. 
During the training process, the proposed ANN captures general relationships between the inputs (i.e. conditions of the examined $\mathrm{ADN}$ ) and the targets (parameters of the equivalent model). Once the training is finished, this information can be used to derive model parameters that simulate adequately new network conditions, i.e. network conditions different from those used for the training of the ANN. To derive the general relationships between network conditions and the model parameters, the ANN iteratively adjusts its weights and biases to minimize the following mean squared error $(\mathrm{mse})$ :

$$
m s e=\frac{1}{R} \sum_{r=1}^{R}(t(r)-y(r))^{2}
$$

$R$ is the total number of elements contained in $\boldsymbol{t}$ and $\boldsymbol{y}$.

An issue that may occur during the training phase is the so-called overfitting problem [33]. In this case, the ANN memorizes the training examples but does not learn to generalize to new inputs. Therefore, fails to predict reliably future observations [33]. To improve the generalization capabilities of the proposed ANN and to avoid overfitting issues, in this paper, the early stopping technique is adopted [33]. For this purpose, as shown in Fig. $4 \mathrm{a}$, the $m$ data sets of the $k$-th cluster are randomly divided into two subsets, containing $m_{k, T R}$ and $m_{k, V}$ data, respectively. The former is the training set and it is used to update weights and biases of the ANN. The second one is the validation set. The mse is monitored during the training process for both the training and the validation data sets. The corresponding mse is denoted as tmse and vmse, respectively. During the initial phase of the training, both the tmse and the vmse decreases. However, when the ANN begins to overfit the training data, the vmse begins to rise. If the validation error increases for a specified number of iterations (six in this paper), the training is terminated and weights and biases are set to the values which correspond to the minimum validation error [33].

Concerning the training of the proposed ANN, a number of critical parameters must be defined [33], [34], i.e. the TF for the hidden and the output layer, the training algorithm and the number of neurons per layer. The TF for the output layer must be the linear (purelin) function to allow outputs to acquire any finite value [33]. On the other hand, the TF for the hidden layer can be either the log-sigmoid (logsig) or the tansigmoid (tansig) function [33], [34]. Concerning the training algorithm, a method compatible with the early stopping technique, must be used [33]. Regarding the number of neurons, in the literature there are no specific guidelines for defining the optimum number [12], [33]. Therefore, to optimally define all the above parameters, a parametric analysis is conducted. The corresponding results are presented in Section IV.

\section{Testing Procedure}

In this phase, the remaining $N_{T}$ data are used to crossvalidate the performance of the derived equivalents. The testing is performed as depicted in Fig. 4b. Initially, the most suitable ANN is selected. For this purpose, testing data are compared with the $K$ centroids using the Euclidean distance
[27]. The selected ANN corresponds to the most similar centroid (i.e. the centroid with the lowest Euclidean distance).

Afterwards, variables $P_{0}, Q_{0}, V_{0}, L M$, and $G M$ of the testing data are forwarded as inputs to the corresponding ANN. Then, the ANN calculates a new set of model parameters for the adopted equivalent model, that describes effectively the corresponding network conditions. This set of parameters is determined based on the general relationships (i.e. the relationships between network conditions and model parameters), which have been derived during the training phase.

Subsequently, the resulting model parameters are used to regenerate the real and reactive power responses of the ADN. To accomplish this, the dynamic responses of voltage are introduced as inputs in the block diagram of Fig. 2. The output of the block diagram contains the estimated real or reactive power responses. The estimated responses are then compared with the actual measurements by means of root mean square error $R M S E$, which is defined as:

$$
R M S E=\sqrt{\frac{1}{T} \sum_{\tau=1}^{T}\left(y_{\text {meas }}(\tau)-y_{\text {est }}(\tau)\right)^{2}}
$$

where $y_{\text {meas }}(\tau)$ and $y_{\text {est }}(\tau)$ are the measured and the estimated responses at sample $\tau$, respectively, and $T$ is the total number of samples.

\section{E. Online Application of the Proposed Method}

A significant advantage of the proposed method is that can be used for online applications, e.g. control room applications. In this case, the following procedure is applied: Initially, the DSO develops the required database. Using this database, the training (i.e. clustering, parameter estimation, training of ANNs) is performed off-line. Once the training is completed, the derived ANNs can be used for online applications.

During the online application, the DSO introduces the operating conditions of the ADN, the load and the generation mix to the corresponding ANN. Subsequently, the ANN provides in close to real-time a set of model parameters, that optimally describe the corresponding network conditions. The DSO can use these equivalents to conduct large-scale simulations to evaluate the dynamic behavior of the ADN under several contingencies and to investigate the interaction of the ADN with the main transmission grid.

\section{Evaluation of the Proposed Method Using LABORATORY MEASUREMENTS}

\section{A. System Under Study}

To validate the applicability of the proposed method a series of experiments were conducted, using the three-phase, $400 \mathrm{~V}$, $50 \mathrm{~Hz}$, laboratory-scale ADN of Fig. 6. The test setup is supplied by a three-phase programmable voltage source (PVS) and consists of two sub-grids. Sub-grid \#1 consists of a 64step $10 \mathrm{~kW} / 7.5 \mathrm{kVar}$ static load bank (SLB), used to emulate the behavior of conventional power system loads [35], a $2 \mathrm{kVA}$ synchronous generator (SG), used to emulate the behavior of distributed synchronous generators [8], as well as a $5.5 \mathrm{kVA}$, 0.87 lagging asynchronous machine (AM1). SG is driven by 


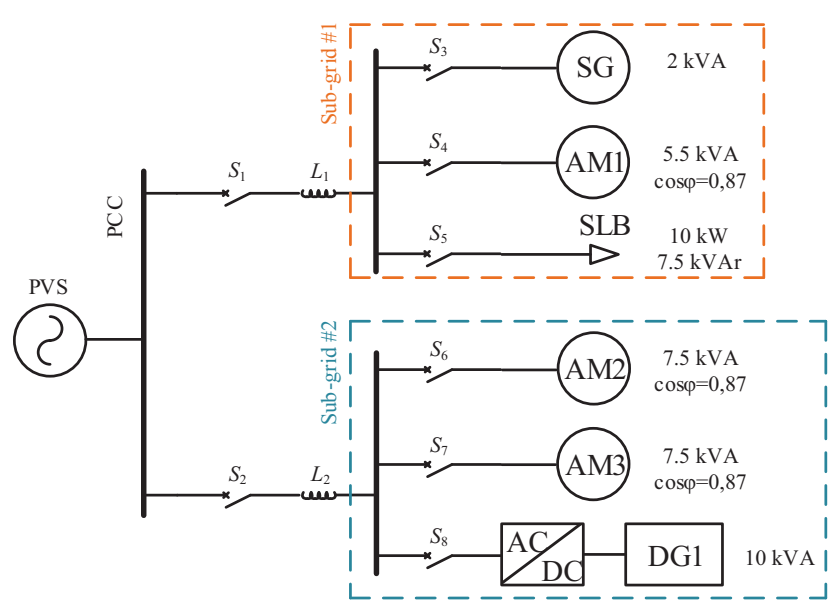

Fig. 6. Laboratory-scale ADN.

a dc motor and follows an active power - frequency $(P$ $f)$, reactive power - voltage $(V-Q)$ droop control scheme. Sub-grid \#2 contains a $10 \mathrm{kVA}$ inverter interfaced distributed generator (DG1), which operates under a constant power (i.e. $P-Q)$ mode, injecting fixed amount of real power. This unit is used in the experimental setup to emulate the behavior of inverter interfaced DGs [8]. In sub-grid \#2 two $7.5 \mathrm{kVA}$, 0.87 lagging asynchronous machines (AM2 and AM3) are also installed. The torque of all induction machines is controllable. Thus, they can operate both as motors or generators. In the former case, induction machines are used to emulate the dynamic behavior of conventional rotating power system loads [35], while in the latter to imitate the behavior of induction generators [36].

A variety of network configurations is examined by switching on and off the switches of the test setup $\left(S_{1}-S_{8}\right)$. Different loading conditions are examined by altering the power of the installed components. To emulate different operating conditions, the voltage level at the PCC ranges between $360 \mathrm{~V}$ (0.9 p.u.) and $440 \mathrm{~V}$ (1.1 p.u.). To investigate system dynamics, voltage disturbances, ranging between -0.1 p.u. and 0.1 p.u. are introduced using the PVS. Dynamic responses of real and reactive powers are calculated by means of the voltage and current at the PCC. The latter responses were recorded at a rate of 500 samples per second using voltage and current transformers, respectively. A detailed description of the measurement infrastructure can be found in [37].

Using this setup, a set of 510 cases, representing different network configurations, loading conditions, and voltage disturbances were generated. The measured data was randomly divided into two separate groups. The first group contains $80 \%$ of the data and is used to derive robust model parameters (i.e. $N_{D}=408$ ). The remaining $20 \%$, i.e. $N_{T}=102$, is used to crossvalidate the performance of the derived models.

\section{B. Training Procedure}

The $N_{D}$ dynamic responses along with the corresponding load and generation mix (represented by variables $L M$ and $G M)$ are forwarded as inputs to the proposed method to derive robust model parameters. $L M$ varies from $0 \%$ to $100 \%$. A value equal to $0 \%$ denotes that the only type of loads installed in the examined ADN is static loads. On the other hand, a value equal to $100 \%$ means that the load of the ADN consists only of asynchronous motors. Variable $G M$ is a three digit numeric string. Each digit can be either 0 or 1 . The first digit is used to denote if inverter-interfaced DGs are connected to the grid (in this case it is equal to 1) or not (the value of the digit is 0 ). The second one is used to represent the presence of asynchronous machines operated as generators, while the third one to denote the presence of synchronous generators.

The WCBCR, as computed using the training data, is depicted in Fig. 7. Based on the knee-point criterion, a number of four clusters, i.e., $K=4$, is used to describe the operating conditions of the examined ADN. The performance of the ANNs is assessed by calculating the corresponding tmse and vmse. For this purpose, a parametric analysis is conducted assuming different number of neurons, different training algorithms and different TFs for the hidden layer of the ANNs. The number of neurons ranges from 5 to 80 , assuming a step equal to 5. Moreover, three training algorithms compatible with the early stopping technique, namely the Levenberg-Marquardt (LM), the resilient back propagation (RP), and the conjugate gradient back propagation (SCG) are examined. Concerning the TF of the hidden layer, the performance of both the logsig and the tansig functions is evaluated. For each combination of neurons, training algorithm and $\mathrm{TF}$, a set of 100 distinct initial conditions for weight and bias matrices are randomly generated by applying the Monte Carlo (MC) method. The mean values of the tmse and the vmse, provided by the $\mathrm{MC}$ simulations, are depicted in Figs. 8 and 9, respectively. The corresponding mean execution times are presented in Fig. 10.

As shown, in all cases the training of the ANNs using the LM algorithm requires higher execution times compared to the cases when the RP and the SCG are used. However, as illustrated in Figs. 8 and 9, the LM algorithm provides the best results, i.e. the minimum values, for both the tmse and the vmse. Additionally, it is clear that the impact of the TF of the hidden layer on the performance of the ANNs is rather limited, since in all cases trivial differences are observed. However, it is interesting to note that the logsig function seems to be more suitable for this specific application, since it generally provides lower values for both the tmse and the vmse compared to the tansig function. Moreover, it is evident that the tmse is generally reduced as the number of neurons increases. This remark is valid for all the examined training algorithms and for both TFs. Concerning the vmse a different behavior is observed. Initially, as the number of neurons

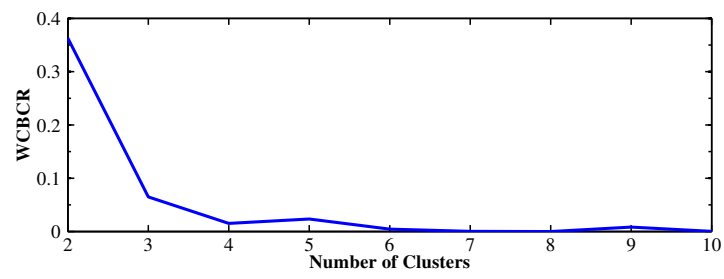

Fig. 7. WCBCR index for different number of clusters. 


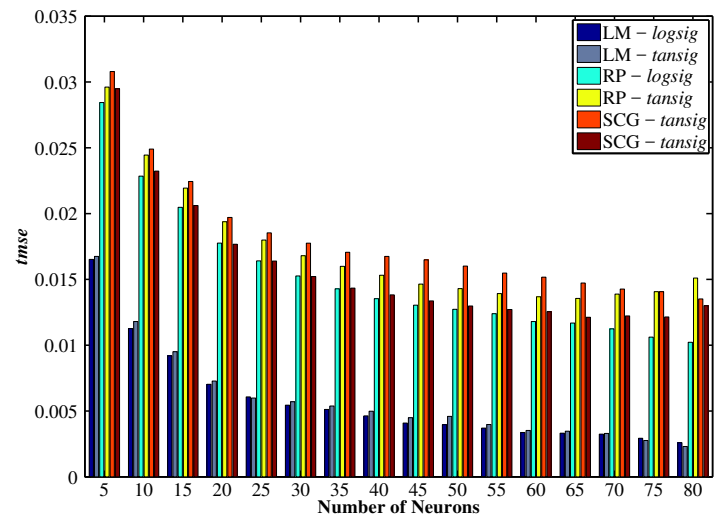

Fig. 8. Impact of several parameters on the tmse.

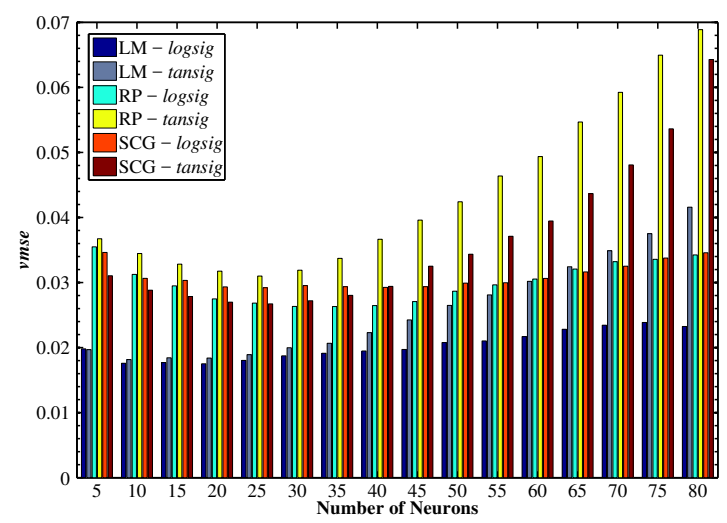

Fig. 9. Impact of several parameters on the vmse.

increases, the vmse decreases. However, after a certain point, a further increase in the number of neurons leads to an increase to the vmse values. This actually implies that the ANN is considerably large and thus overfits the training data [33].

The minimum vmse is observed for a number of 10 neurons, when the logsig function is used for the activation of the hidden layer and the training is performed via the LM. Hence, these settings are selected for the training of the ANNs. In this case, tmse is merely 0.0113 and the training procedure requires less than $2 \mathrm{~s}$. The generalization capabilities of the proposed method are further evaluated in the next subsection.

\section{Testing Procedure}

The accuracy of the proposed method is evaluated here using the testing data $\left(N_{T}\right)$. Additionally, its performance is compared with a conventional approach in which statistical analysis is applied to each cluster and representative parameters are computed by means of the corresponding mean values [10]. The execution time, required from the proposed method to derive robust model parameters for the $N_{T}$ data sets, was in all cases lower that $0.1 \mathrm{~s}$. This low execution time verifies the applicability of the method for close to real-time applications.

Representative instances of the undertaken tests are presented in Figs. 11a - 11h. More specifically, in these Figs., the real and reactive power responses, estimated by the proposed and the conventional approach, are compared with the corresponding laboratory measurements. The proposed method simulates more accurately compared to the conventional approach

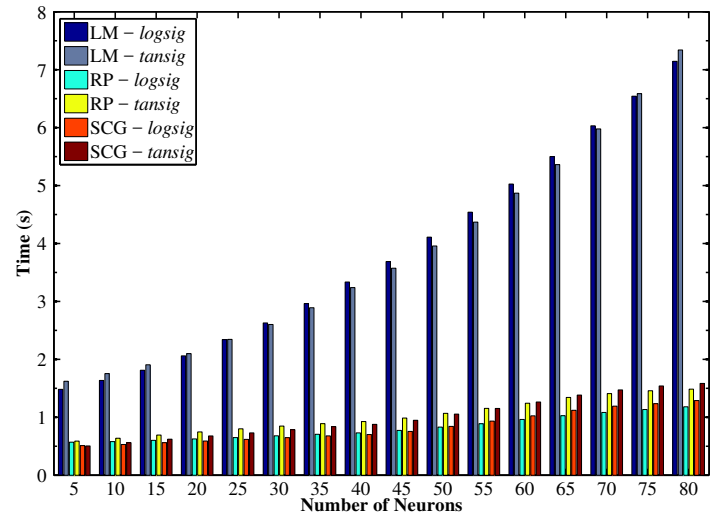

Fig. 10. Impact of several parameters on the required execution time.

the dynamic behavior of both real and reactive power. Indeed, using the proposed method, the new steady-state and the undershoot/overshoot of real and reactive power are accurately simulated in all cases. Additionally, oscillatory responses, as the one presented in Fig. 11c, are efficiently modeled. Finally, as shown in Fig. 11e, reverse power flow phenomena that may occur during system disturbances are accurately simulated.

To provide a further insight in the accuracy of the proposed and the conventional approach, the probability density functions (PDF) and the cumulative distribution functions (CDF) of the RMSEs for the $N_{T}$ data set are presented in Fig. 12. Concerning the modeling of the real power, PDFs indicate that the RMSE is most likely to be about 0.008 for the proposed method and 0.016 for the conventional approach. Additionally, CDFs reveal that $90 \%$ of the resulting RMSEs are under 0.039 for the proposed approach, while the corresponding percentage for the conventional approach is equal to $65 \%$. Similar results are also observed for the modeling of the reactive power. More specifically, 90\% of the resulting RMSEs for the proposed approach are lower than 0.021 . On the other hand, the corresponding percentage for the conventional approach is merely $21 \%$. The maximum RMSE of the proposed approach for the modeling of the reactive power is equal to 0.041 . Using the conventional approach, $45 \%$ of the testing data result in higher RMSEs compared to this value. Finally, PDFs reveal that the RMSE is most likely to be about 0.011 for the proposed method and 0.034 for the conventional approach.

\section{Further Investigations}

In this Subsection the impact of several parameters on the accuracy of the proposed method is investigated. Initially, the impact of load and generation mix (parameters $L M$ and $G M$, respectively) is evaluated. As discussed in Section III, these parameters can be assessed through forecasts or smart meter recordings. In case such data is not available or is missing, it is expected that the DSO will not be able to determine $L M$ and GM. Therefore, in this Subsection, the training of the proposed method is evaluated by neglecting these two variables. To accomplish this, the last two rows of the input matrix, i.e. $\boldsymbol{x}$, are erased. The resulting PDFs and CDFs of the RMSEs for the testing data set are presented in Fig. 12. As shown, neglecting $L M$ and $G M$ leads to noticeable 

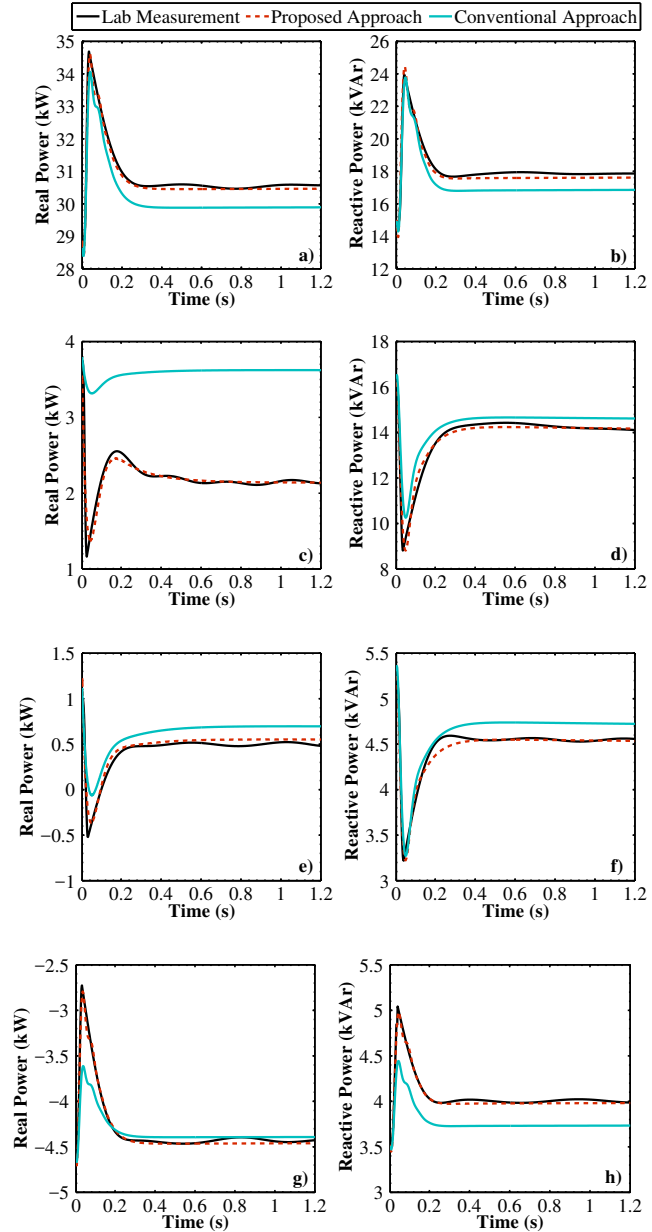

Fig. 11. Indicative responses. i) The ADN imports real power. A step-up voltage disturbance is examined. Modeling of a) real and b) reactive power. ii) The ADN imports real power. A step-down voltage disturbance is examined. Modeling of c) real and d) reactive power. iii) The ADN imports real power A step-down voltage disturbance is examined. Bi-directional power flow is observed during the disturbance. Modeling of e) real and $\mathrm{f}$ ) reactive power. iv) The ADN exports real power. A step-up voltage disturbance is examined. Modeling of $\mathrm{g}$ ) real and $\mathrm{h}$ ) reactive power.

degradation in the performance of the proposed method. Nevertheless, even in this case, the equivalent models, derived using the proposed method, are more accurate compared to the equivalents developed using the conventional approach.

The impact of the clustering technique on the accuracy of the proposed method is also evaluated. Towards this objective, the $k$-means++ algorithm is compared with the $k$-medoids and the fuzzy $c$-means algorithm. To provide a common comparative base, in all cases, a number of four clusters is considered. The resulting CDFs are depicted in Fig. 13. Based on these results, it is evident that the impact of the clustering method on the accuracy of the method is rather limited, since in all cases trivial differences are observed.

\section{DISCUSSION AND CONCLUSIONS}

Parameters of measurement-based equivalent models can be updated only when new disturbances are available. Due to this inherent limitation, the accuracy of these models for online applications is rather limited. To address this issue, in this paper, a new method, based on artificial-intelligence
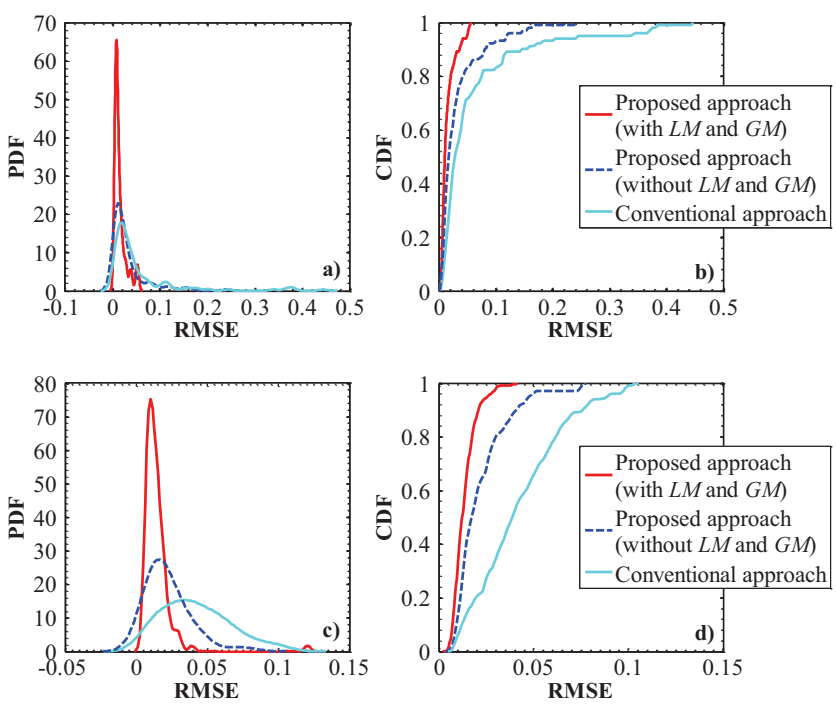

Fig. 12. Modeling of real power. a) PDFs and b) CDFs. Modeling of reactive power. c) PDFs and d) CDFs.
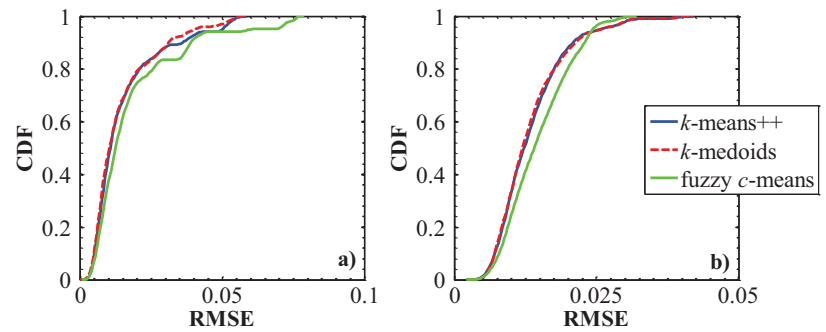

Fig. 13. CDFs of the proposed method, assuming different clustering techniques. a) Modeling of real and b) reactive power.

techniques, is developed. In the proposed framework, a training data set, containing disturbance events, is used for the estimation of the required model parameters. Using the derived parameters, ANNs are trained. The ANNs aim to identify general relationships between the parameters of the adopted equivalent model and the pre-disturbance operating conditions, the load and the generation mix of the examined ADN.

During the online application, operating conditions of the ADN as well as the load and the generation mix are forwarded as inputs to the developed ANNs, which provide (without requiring new disturbance events) in close to real-time sets of model parameters that optimally describe the examined ADN.

The effectiveness of the proposed method is evaluated using measurements acquired from a laboratory-scale ADN, while its performance is compared with a conventional approach. Comparison results reveal that the proposed method presents superior performance compared to the conventional one, simulating more accurately the complex dynamic phenomena that occur in ADNs (i.e. oscillations, bi-directional power flows).

Furthermore, the impact of several parameters on the accuracy of the proposed method is thoroughly investigated. For this purpose, a parametric analysis is conducted to determine the TF for the hidden layer of the ANNs, the most suitable training algorithm, and the optimal number of neurons. The analysis shows that the logsig function is more suitable compared to the tansig, since it provides lower errors, while the Levenberg-Marquardt is the most appropriate algorithm for 
the training of the ANNs. Moreover, the conducted analysis reveals that a number of 10 neurons can ensure very accurate results. The above settings can be used as indicative values for the derivation of generic aggregated equivalent models.

Additionally, the impact of the clustering technique as well as the impact of load and generation mix on the accuracy of the proposed method are evaluated. Results indicate that the accuracy of the proposed method is not affected by the clustering technique used. Moreover, evaluation results reveal that input of information concerning load and generation mix of the examined ADN can enhance the accuracy of the proposed method. Nevertheless, the proposed method results in more accurate and robust models as compared to the conventional approach, even in cases where this information is not available.

Based on the evaluation results, it can be concluded that the proposed method constitutes a reliable tool that can be used from DSOs for the derivation of generic measurement-based dynamic equivalent models.

Future work will incorporate load and generation mix estimates of the ADN using measurements acquired at PCC with the external grid. This way, the impact of forecast errors on the accuracy of the proposed method will be eliminated.

\section{REFERENCES}

[1] S. M. Zali and J. V. Milanovic, "Generic model of active distribution network for large power system stability studies," IEEE Trans. Power Syst., vol. 28, no. 3, pp. 3126-3133, Aug., 2013.

[2] J. V. Milanovic and S. M. Zali, "Validation of equivalent dynamic model of active distribution network cell," IEEE Trans. Power Syst., vol. 28, no. 3, pp. 2101-2110, Aug., 2013.

[3] Cigre Report, Working Group C4.605. Modelling and aggregation of loads in flexible power networks, Feb. 2014

[4] J. V. Milanovic and M. Kayikci, "Transient responses of distribution network cell with renewable generation," in 2006 IEEE PES Pow. Syst. Conf. and Exp., Oct., 2006, pp. 1919-1925.

[5] Z. Miao, A. Domijan, and L. Fan, "Investigation of microgrids with both inverter interfaced and direct ac-connected distributed energy resources," IEEE Trans. Power Del., vol. 26, no. 3, pp. 1634-1642, Jul. 2011.

[6] F. Katiraei, M. R. Iravani, and P. W. Lehn, "Small-signal dynamic model of a micro-grid including conventional and electronically interfaced distributed resources," IET Gen., Trans. Dist., vol. 1, no. 3, pp. 369 378, May 2007.

[7] U. D. Annakkage, et al., "Dynamic system equivalents: A survey of available techniques," IEEE Trans. Power Del., vol. 27, no. 1, pp. 411420, Jan. 2012.

[8] P. N. Papadopoulos, et al., "Black-box dynamic equivalent model for microgrids using measurement data," IET Gen., Trans. Dist., vol. 8, no. 5, pp. 851-861, May 2014.

[9] B.-K. Choi, H.-D. Chiang, Y. Li, H. Li, Y.-T. Chen, D.-H. Huang, and M. G. Lauby, "Measurement-based dynamic load models: derivation, comparison, and validation," IEEE Trans. Power Syst., vol. 21, no. 3, pp. 1276-1283, Aug., 2006.

[10] D. P. Stojanovic, L. M. Korunovic, and J. Milanovic, "Dynamic load modelling based on measurements in medium voltage distribution network," Elect. Power Syst. Res., vol. 78, no. 2, pp. 228 - 238, Feb., 2008.

[11] Measurement-Based Load Modeling. EPRI, Palo Alto. CA: 2006. 1014402.

[12] K. S. Metalinos, T. A. Papadopoulos, and C. A. Charalambous, "Derivation and evaluation of generic measurement-based dynamic load models," Elect. Power Syst. Res., vol. 140, pp. 193-200, 2016.

[13] H. Renmu, M. Jin, and D. J. Hill, "Composite load modeling via measurement approach," IEEE Trans. Power Syst., vol. 21, no. 2, pp. 663-672, May 2006.

[14] E. O. Kontis, T. A. Papadopoulos, A. I. Chrysochos, and G. K. Papagiannis, "Measurement-based dynamic load modeling using the vector fitting technique," IEEE Trans. Power Syst., vol. 33, no. 1, pp. 338-351, Jan., 2018.
[15] E. O. Kontis, A. I. Chrysochos, G. K. Papagiannis, and T. A. Papadopoulos, "Development of measurement-based generic load models for dynamic simulations," in 2015 IEEE Eindhoven PowerTech, Jun., 2015, pp. 1-6.

[16] A. M. Azmy, I. Erlich, and P. Sowa, "Artificial neural network-based dynamic equivalents for distribution systems containing active sources," IET Gener. Transm. Dis., vol. 151, no. 6, pp. 681-688, Nov., 2004.

[17] C. Changchun, et al., "Micro-grid dynamic modeling based on RBF Artificial Neural Network," in International Conference on Power System Technology, Oct. 2014, pp. 3348-3353.

[18] A. Arif, et al., "Load Modeling-A Review," IEEE Trans. Smart Grid, vol. 9, no. 6, pp. 5986-5999, Nov. 2018.

[19] H. Golpira, H. Seifi, and M. R. Haghifam, "Dynamic equivalencing of an active distribution network for large-scale power system frequency stability studies," IET Gen., Trans. Dist, vol. 9, no. 15, pp. 2245-2254, Nov. 2015.

[20] E. O. Kontis, I. S. Skondrianos, T. A. Papadopoulos, A. I. Chrysochos, and G. K. Papagiannis, "Generic dynamic load models using artificial neural networks," in 2017 52nd Int. Univ. Power Eng. Conf. (UPEC), Aug., 2017, pp. 1-6.

[21] D. Karlsson and D. J. Hill, "Modelling and identification of nonlinear dynamic loads in power systems," IEEE Trans. Power Syst., vol. 9, no. 1, pp. 157-166, Feb., 1994.

[22] E. O. Kontis, S. P. Dimitrakopoulos, A. I. Chrysochos, G. K. Papagiannis, and T. A. Papadopoulos, "Dynamic equivalencing of active distribution grids," in 2017 IEEE Manchester PowerTech, Jun., 2017, pp. $1-6$.

[23] E. O. Kontis, T. A. Papadopoulos, A. I. Chrysochos, and G. K. Papagiannis, "On the applicability of exponential recovery models for the simulation of active distribution networks," IEEE Trans. Power Del., in press.

[24] B. Stephen and S. J. Galloway, "Domestic load characterization through smart meter advance stratification," IEEE Trans. Smart Grid, vol. 3, no. 3, pp. 1571-1572, Sept., 2012.

[25] J. Liang, S. K. K. Ng, G. Kendall, and J. W. M. Cheng, "Load signature study - part i: Basic concept, structure, and methodology," IEEE Trans. Power Del., vol. 25, no. 2, pp. 551-560, Apr. 2010.

[26] J. V. Milanović and Y. Xu, "Methodology for estimation of dynamic response of demand using limited data," IEEE Trans. Power Syst. vol. 30, no. 3, pp. 1288-1297, May 2015.

[27] I. P. Panapakidis, "Clustering based day-ahead and hour-ahead bus load forecasting models," Int. J. Electr. Power Energy Syst., vol. 80, pp. 171178, 2016.

[28] S. M. Zali, N. C. Wooley, and J. V. Milanovic, "Developemnt of equivalent dynamic model of distribution network using clustering procedure," in 17th Power Syst. Computation Conf., Jun., 2011, pp. 1-8.

[29] D. Issicaba, A. S. Costa, and J. L. Colombo, "Real-time monitoring of points of common coupling in distribution systems through state estimation and geometric tests," IEEE Trans. Smart Grid, vol. 7, no. 1, pp. 9-18, Jan., 2016.

[30] G. J. Tsekouras, N. D. Hatziargyriou, and E. N. Dialynas, "Twostage pattern recognition of load curves for classification of electricity customers," IEEE Trans. Power Syst., vol. 22, no. 3, pp. 1120-1128, Aug. 2007.

[31] B. Gustavsen, "Improving the pole relocating properties of vector fitting," IEEE Trans. Power Del., vol. 21, no. 3, pp. 1587-1592, Jul. 2006.

[32] D. J. Trudnowski, J. M. Johnson, and J. F. Hauer, "Making Prony analysis more accurate using multiple signals," IEEE Trans. Power Syst., vol. 14, no. 1, pp. 226-231, Feb. 1999.

[33] H. Demuth and M. Beale, MATLAB Neural Network Toolbox User's Guide. Version 4.

[34] Y. Xu and J. V. Milanović, "Artificial-intelligence-based methodology for load disaggregation at bulk supply point," IEEE Trans. on Power Syst., vol. 30, no. 2, pp. 795-803, Mar, 2015.

[35] "Load representation for dynamic performance analysis (of power systems)," IEEE Trans. Power Syst., vol. 8, no. 2, pp. 472-482, May 1993.

[36] J. Liu and C. Chu, "Long-term voltage instability detections of multiple fixed-speed induction generators in distribution networks using synchrophasors," IEEE Trans. Smart Grid, vol. 6, no. 4, pp. 2069-2079, Jul. 2015.

[37] A. J. Roscoe, A. Mackay, G. M. Burt, and J. R. McDonald, "Architecture of a network-in-the-loop environment for characterizing ac powersystem behavior," IEEE Trans. on Ind. Electron., vol. 57, no. 4, pp. 1245-1253, Apr. 2010. 